Original Research Paper

\title{
Persian Curve Versus Monte Carlo Method
}

\author{
1*Abdolrasoul Ranjbaran, ${ }^{2}$ Mohammad Ranjbaran and ${ }^{3}$ Fatema Ranjbaran \\ ${ }^{1}$ Department of Civil and Environmental Engineering, Shiraz University, Shiraz, Iran \\ ${ }^{2}$ Department of Chemical Engineering, Yasuj University, Yasuj, Iran \\ ${ }^{3}$ Department of Mechanical Engineering, Shiraz University, Shiraz, Iran
}

\author{
Article history \\ Received: 20-05-2021 \\ Revised: 08-06-2021 \\ Accepted: 15-06-2021 \\ Corresponding Author: \\ Abdolrasoul Ranjbaran \\ Department of Civil and \\ Environmental Engineering, \\ Shiraz University, Iran \\ E-mail: ranjbarn@shirazu.ac.ir \\ aranjbaran@yahoo.com
}

\begin{abstract}
The Monte Carlo Simulation method is a powerful tool for solving problems including random variables. The basic idea to implement a Monte Carlo simulation is to first generate samples of random inputs from their assumed distribution functions and then perform a deterministic calculation on the generated random inputs, based on mathematical modeling of the system, to obtain output results. An early version of Monte Carlo simulation is the famous needle experiment. The idea of random experiment, have been used for solving many complex problems. Simulation based approaches have some disadvantages. Its implementation needs a massive use of computational resource and long calculation times. Moreover, providing linkage between input to the system and its output is difficult. Toward remedy, the phenomenon is considered as the change in the state of the system. Via logical reasoning, concise mathematics and using real world data, the output is related to the input via the Persian Curve. The Persian Curve provided a simple, cheap and exact solution to the problem. Consequently the Persian Curve is proposed as a replacement for the Monte Carlo Simulation. The validity of the work is verified via concise mathematics and comparison of the results with those of the others.
\end{abstract}

Keyword: Monte Carlo Simulation, Persian Curve, Change of State Philosophy, State Functions, Probability Density Function, Expected Value, Random Variable

\section{Introduction}

The Monte Carlo Simulation (MCS) is a well-known statistical method which is used for analysis of phenomena with no clear deterministic solution, Fig. 1. Despite of its success in different field of human knowledge, the (MCS) is an expensive, time consuming and uncertain method (Badger, 1994; Veach, 1998; Bonate, 2001; Laosiritaworn, 2002; Lefebvre, 2007; Murray, 2007; Manohar, 2009; Wijesinghe, 2011; Du, 2012; Bolin, 2013; Cook et al., 2013; Parkinson, 2013; Pollock, 2013; Romano, 2013; Zio, 2013; Goerdin, 2014; Poole, 2014; Rawlinson, 2015; Hahn, 2015; Wang, 2015; Hochuli, 2016; Sánchez, 2016; Zhao, 2016; Fadele, 2017; Feng, 2017; Mouawad, 2017; Schwarm, 2017a; 2017b; Haqiqat and Müller, 2018; Hou, 2018; Laengen, 2018; Albes, 2019; Huda, 2018; Pakyuz-Charrier, 2018; Unwin, 2018; Wang, 2018; Webster, 2019; Zhang, 2019; Mazhdrakov et al., 2018; Corbella, 2019; de Freitas, 2019; Berg, 2019; Alamri, 2020; Apostolopoulou, 2019; Bhatia, 2020; Cumberworth,
2021; Ead, 2020; Ketron, 2020; Cosgrove, 2020; Dash, 2020; Debrot, 2020; Diniz, 2020; Eagle, 2020; Guijarro Gámez, 2020; Nilakanta, 2020; Welding, 2020; Wang, 2021; Sheridan-Methven, 2021; Sheridan-Methven, 2020). The Abdolrasoul Ranjbaran Team (ART) conducted an intensive research, for analysis of natural phenomena, in the past two decades in the Shiraz University, Iran (Ranjbaran et al., 2008; Ranjbaran and Rousta, 2009; Ranjbaran, 2010; Ranjbaran et al., 2011; Ranjbaran, 2012; Ranjbaran and Rousta, 2013a; 2013b; Ranjbaran et al., 2013a; 2013b; Ranjbaran and Ranjbaran, 2014; Ranjbaran, 2015; Ranjbaran and Ranjbaran, 2016; 2017a; 2017b; 2017c; 2018; Ranjbaran et al., 2020a-f; 2021a; 2021b). The result of her research is digested in the Persian Curve (PC), Fig. 2. The aim of the presented paper is to show that the (PC) is a good and logical alternative for the (MCS) in analysis of natural phenomena. The content of the paper is managed as follows. The basic principles of the (MCS) is expressed first. The study is continued with logical derivation of the (PC) basics. 


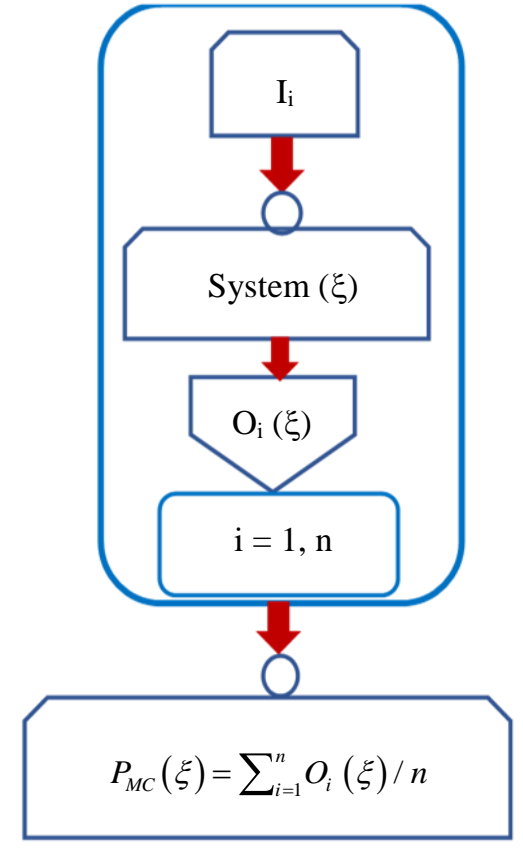

Fig. 1: Monte carlo simulation model

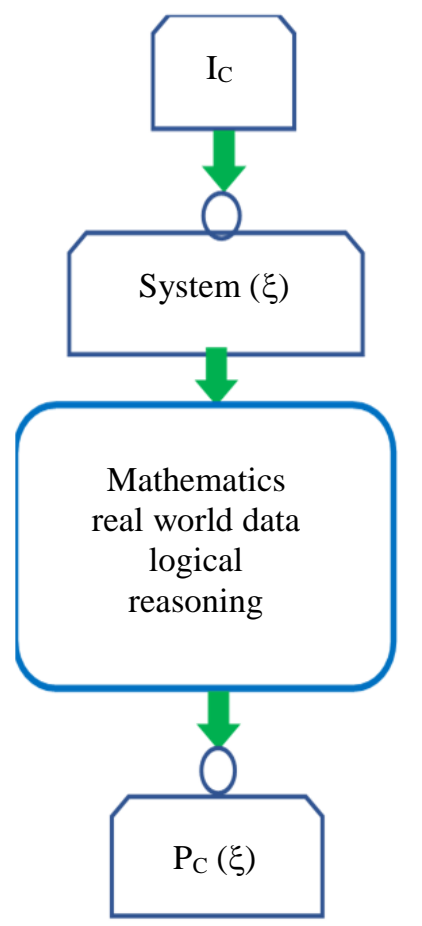

Fig. 2: Persian curve model

The work is continued with verification via analysis of fragility curve for structures, conclusions and list of references. In this study a phenomenon is considered as change in the state of a system. The state variable $(\xi \in$
$[0,1])$ is defined as the lifetime or identification parameter of systems. Every system is expressed by its specific $(\xi)$. Consequently all derivations are finally expressed in terms of the $(\xi)$.

\section{The Monte Carlo Simulation}

The Monte Carlo Simulation is a powerful tool for analysis of complex systems via including random variables (Badger, 1994; Veach, 1998; Bonate, 2001; Laosiritaworn, 2002; Lefebvre, 2007; Murray, 2007; Manohar, 2009; Wijesinghe, 2011; Du, 2012; Bolin, 2013; Cook et al., 2013; Parkinson, 2013; Pollock, 2013; Romano, 2013; Zio, 2013; Goerdin, 2014; Poole, 2014; Rawlinson, 2015; Hahn, 2015; Wang, 2015; Hochuli, 2016; Sánchez, 2016; Zhao, 2016; Fadele, 2017; Feng, 2017; Mouawad, 2017; Schwarm, 2017a; 2017b; Haqiqat and Müller, 2018; Hou, 2018; Laengen, 2018; Albes, 2019; Huda, 2018; Pakyuz-Charrier, 2018; Unwin, 2018; Wang, 2018; Webster, 2019; Zhang, 2019; Mazhdrakov et al., 2018; Corbella, 2019; de Freitas, 2019; Berg, 2019; Alamri, 2020; Apostolopoulou, 2019; Bhatia, 2020; Cumberworth, 2021; Ead, 2020; Ketron, 2020; Cosgrove, 2020; Dash, 2020; Debrot, 2020; Diniz, 2020; Eagle, 2020; Guijarro Gámez, 2020; Nilakanta, 2020; Welding, 2020; Wang, 2021; Sheridan-Methven, 2021; Sheridan-Methven, 2020). The basic idea to implement a Monte Carlo Simulation is to first generate samples of random inputs from their assumed distribution functions. Then perform a deterministic calculation on the generated random inputs, based on mathematical modeling of the system, to obtain numerical results. An early version of Monte Carlo Simulation is the famous needle experiment, performed by the French mathematician Comte de Buffon (1707-1788). Consider a plane with parallel lines distanced at (d) and a needle, with a length $(\mathrm{L}<\mathrm{d})$ that is randomly positioned on the plane. Note that: (1) The shortest distance from the needle center to the line $(x)$, is uniformly distributed over $(0$, $d / 2$ ). (2) The angle between the needle and a line $(\theta)$ also follows a uniform distribution over $(0, \pi / 2)$. (3) the needle crosses a line when $(x \leq L \sin \theta / 2)$. The two random variables ( $x$ and $\theta$ ) are independent. Therefore, their joint probability density function is $(f(x, \theta)=$ $4 / \pi d)$. Let (A) denote the event that the needle lies across a line. The probability of $(P(A)=2 L / \pi d)$ is determined in Eq. (1) as follows:

$$
P(A)=\int_{0}^{\frac{\pi}{2}}\left(\int_{0}^{\frac{L}{2} \sin (\theta)} f(x, \theta) d x=\frac{L}{2}|\sin (\theta)| \frac{4}{\pi d} d \theta\right)
$$


Buffon verified this probability by eventually throwing a needle on the plane with parallel lines. This experiment, reflects the basic idea of Monte Carlo Simulation. That is, performing the experiment $(n)$ times, if the needle crosses a line $(\mathrm{m})$ times, then the probability of $(A)$ is approximated in Eq. (2):

$$
P(A)=m / n
$$

As marked by Laplace in 1812 , one can estimate the value of $(\pi)$ by conducting the Buffon's needle experiment and setting Eq. (1) equal to Eq. (2), in Eq. (3):

$m / n=2 L / \pi d \rightarrow \pi=2 n L / m d$

In 1901, the Italian mathematician Lazzarini (Badger, 1994) conducted the needle experiments and obtained an estimate of the $(\pi)$ to six significant digits, as in Eq. (4):

$$
(d ; L ; n ; m)=(d ; 5 d / 6 ; 3480 ; 1808)
$$$$
\pi=3.14159292
$$

This experiment offered such an impression that one can estimate a probability of an event or a random quantity via random simulation. In 1946, the physicist from Los Alamos were working on the distance likely to be traveled by the neutron in different materials under the Manhattan Project. They were unable to solve the problem using conventional deterministic mathematical methods. Then Stainislaw Ulam proposed an idea of using random experiments. This idea was subsequently developed by von Neumann, Metropolis and others to solve many complex problems in making the atomic bomb. Since the work was secret, the random experiment method required a code name. Metropolis suggested the name of Monte Carlo, which refers to the Monte Carlo Casino in Monaco where Ulam's uncle borrow money from relatives to gamble (Zhang, 2019). More scientific basis of the Monte Carlo simulation, is the strong law of numbers, which guarantees that the average of a set of independent and identically distributed random variables converges to the mean value with probability 1 .

The strong law of large numbers is expressed as follows. For a sequence of statistically $(n)$ independent and identically distributed random variables $\left(X_{i}, i=1, n\right)$ with a mean value of $(\mu)$, then the average of the variables converges to $(\mu)$ with probability 1 , as in Eq. (5):

$$
\sum_{i=1}^{n} O_{i} / n \rightarrow \mu \text { as } n \rightarrow \infty
$$

Monte Carlo simulation model is shown in Fig. 1. According to the strong law of large numbers, the average converges to certain value as $(n \rightarrow \infty)$. Equation (5), for a system with the characteristics function $(h(\xi, x))$ and the random system output $\left(x=\left(x_{1}, \ldots, x_{n}\right)\right)$ with the probability distribution $\left(f_{o}(x)\right)$ is expressed in Eq. (6) for the system $(\xi)$ :
$P_{M C}(\xi)=\int_{-\infty}^{+\infty} h(\xi, O) f_{O}(O) d O$

The idea of random experiment, have been used for solving many complex problems (Badger, 1994; Veach, 1998; Bonate, 2001; Laosiritaworn, 2002; Lefebvre, 2007; Murray, 2007; Manohar, 2009; Wijesinghe, 2011; Du, 2012; Bolin, 2013; Cook et al., 2013; Parkinson, 2013; Pollock, 2013; Romano, 2013; Zio, 2013; Goerdin, 2014; Poole, 2014; Rawlinson, 2015; Hahn, 2015; Wang, 2015; Hochuli, 2016; Sánchez, 2016; Zhao, 2016; Fadele, 2017; Feng, 2017; Mouawad, 2017; Schwarm, 2017a; 2017b; Haqiqat and Müller, 2018; Hou, 2018; Laengen, 2018; Albes, 2019; Huda, 2018; Pakyuz-Charrier, 2018; Unwin, 2018; Wang, 2018; Webster, 2019; Zhang, 2019; Mazhdrakov et al., 2018; Corbella, 2019; de Freitas, 2019; Berg, 2019; Alamri, 2020; Apostolopoulou, 2019; Bhatia, 2020; Cumberworth, 2021; Ead, 2020; Ketron, 2020; Cosgrove, 2020; Dash, 2020; Debrot, 2020; Diniz, 2020; Eagle, 2020; Guijarro Gámez, 2020; Nilakanta, 2020; Welding, 2020; Wang, 2021; Sheridan-Methven, 2021; Sheridan-Methven, 2020). Simulation based approaches have some disadvantages. Its implementation needs a massive use of computational resource and long calculation times. Moreover, providing linkage between input to the system and its output via a closed form solution is difficult. In view of Eq. (6) the aim is determination of the $\left(\mathrm{P}_{\mathrm{MC}}\right)$, which is only a function of $(\xi)$ ! There is no sign of the method of solution in it. It appears that using the (MCS) for this job is the worst method that could be selected. Despite of this much of efforts, since the infinity is out of reach then the solution by the (MCS) always contains epistemic uncertainty which is added to the aleatory one. Toward remedy, the Abdolrasoul Ranjbaran Team (ART), conducted an extensive research toward analysis of change in the systems, called the Change of State Philosophy (CSP). The result of her research is the Persian Curve (PC) which is expressed in the next section. As will be observed in the next section, the (ART) investigations, via logical reasoning, concluded directly to the Persian Curve $\left(P_{C}(\xi)\right)$, which clearly can be used in place of $\left(P_{M C}(\xi)\right)$ ! As will be seen, the Persian Curve is equal to the expected value of the system output (obtained by stochastics methods including the (MCS) for $n \rightarrow \infty$ ) with probability 1 . All methods in human knowledge are approximate (since $n<<\infty$ ) as compared to the Persian Curve.

\section{The Persian Curve Basics}

For a change in a system (e.g., structure) the survived capacity and the fled capacity are important (Ranjbaran et al., 2008; Ranjbaran and Rousta, 2009; Ranjbaran, 2010; Ranjbaran et al., 2011; Ranjbaran, 2012; Ranjbaran and 
Rousta, 2013a; 2013b; Ranjbaran et al., 2013a; 2013b; Ranjbaran and Ranjbaran, 2014; Ranjbaran, 2015; Ranjbaran and Ranjbaran, 2016; 2017a; 2017b; 2017c; 2018; Ranjbaran et al., 2020a-f; 2021a; 2021b). The capacity $\left(k_{S}\right)$, called system stiffness, is defined as a positive entity which has a finite specified value at the beginning of the lifetime (origin) and reduces to zero at the end of systems lifetime (destination). The inverse of the system stiffness is called system flexibility $\left(f_{S}\right)$. The concept of stiffness and flexibility are used for better understanding! They are general and are not necessarily those used in the structural mechanics! For a system with a given lifetime, the survived stiffness $\left(k_{S S}=k_{S}-k_{C}\right)$ and the survived flexibility $\left(f_{S S}\right.$ $\left.=f_{S}+f_{C}\right)$ are obviously inverse of each other, where $\left(k_{C}\right)$ is the change stiffness and $\left(f_{C}\right)$ is the change flexibility. This obvious relation is defined in Eq. (7) and shown in Fig. 3 and is used for reliable analysis of changing systems as follows:

$$
\left(k_{S}-k_{C}\right)\left(f_{S}+f_{C}\right)=1
$$

Note that although the $\left(k_{S}\right.$ and $\left.f_{S}\right)$ are known at the origin, the $\left(k_{C}\right.$ and $\left.f_{C}\right)$ at each point along the lifetime should be determined. This is done as follows.

There is only one Eq. (7) at hand for solution. Therefore, for the time being, let the $\left(k_{S}, f_{S}\right.$ and $\left.f_{C}\right)$ as known and solve Eq. (7) for $\left(k_{S S}\right.$ and $\left.k_{C}\right)$ in Eq. (8). Where $(-;-)$ denotes a vector:

$\left(k_{S S} ; k_{C}\right)=\left(S_{R} ; F_{R}\right) k_{S}$

In Eq. (8) the phenomenon functions (the survived function $\left(S_{R}\right)$ and the fled function $\left(F_{R}\right)$ ) are defined in Eq. (9):

$$
\left(S_{R} ; F_{R}\right)=\left(f_{S} ; f_{C}\right) /\left(f_{S}+f_{C}\right)
$$

Note that the phenomenon functions are ratios in a unit interval. Since construction of functionals in terms of two functions is not possible, then the phenomenon functions are customized to the state functions as follows.

For $\left(f_{S}=1\right)$, the phenomenon functions are renamed as the state functions (collection of the Origin function (O) and the destination function (D)) and the state Ratio (R) in Eq. (10):

$$
\left(f_{S} ; f_{C} ; S_{R} ; F_{R}\right)=(1 ; R ; O ; D)
$$

In view of Eq. (10), the state functions are defined as ratios in unit intervals in Eq. (11):

$$
(O ; D)=(1 ; R) /(1+R)
$$

Eq. (11) may be rewritten as boundary value problems in Eq. (12), in which (min and max) denote (minimum and maximum) respectively:
$(O ; D)=\mid \begin{aligned} & (\max ; \min )=(1 ; 0), @ R=0 \\ & (\min ; \max )=(0 ; 1), @ R=\infty\end{aligned}$

Since, from Eq. (11) the state ratio $(R=D / O)$ is defined in a semi-infinite interval $(R \in[0, \infty])$ and it is a function, then it cannot be used as an independent variable, Fig. 4 ! Toward remedy, a state variable in a unit interval $(\xi \in[0,1])$, with a zero value at the origin $(\xi=0)$ and a one value at the destination $(\xi=1)$ is defined. In view of the artifice used and in terms of the state variable, Eq. (12) is rewritten as Eq. (13):

$(O ; D)=\mid \begin{aligned} & (\max ; \min )=(1 ; 0), @ \xi=0 \\ & (\min ; \max )=(0 ; 1), @ \xi=1\end{aligned}$

Make use of the expertise of the authors the state functions are defined as explicit functions of the $(\xi)$, in Eq. (14) where each function is an averages of a polynomial- and a trigonometric-function, as in Eq. (15), Fig. 5:

$$
\begin{aligned}
& 4 O=\left(2+1-6 \xi^{2}+4 \xi^{3}+\cos \pi \xi\right) \\
& 4 D=\left(2-1+6 \xi^{2}-4 \xi^{3}-\cos \pi \xi\right) \\
& 4 O=\left(1+1-6 \xi^{2}+4 \xi^{3}\right)+(1+\cos \pi \xi) \\
& 4 D=\left(1-1+6 \xi^{2}-4 \xi^{3}\right)+(1-\cos \pi \xi)
\end{aligned}
$$

At this stage, attention is paid to enhance the construction of the phenomenon functions. Via the definition of the $\left(k_{S S}\right.$ and $\left.f_{S S}\right)$ in Eq. (7) and concept of crack compliance $\left(f_{C}\right)$ in fracture mechanics (Anderson, $2017)$, the authors detected a fact that, the $\left(f_{C}\right)$ is directly proportional to the $\left(k_{S}\right)$. This detection is called, the Persian Principle of Change (PPC). In view of the (PPC) the $\left(f_{C}\right)$ is innovatively defined in Eq. (16):

$\left(f_{C} / R ; f_{C}\right)=k_{S} / 1 ; k_{S} D / O$

Substitution of Eq. (16) into Eq. (9), concluded into the generalized definition for the phenomenon functions in Eq. (17).

$\left(S_{R} ; F_{R}\right)=\left(O ; k_{S}^{2} D\right) /\left(O+k_{S}^{2} D\right)$

The $\left(k_{S}\right)$ can not be directly determined from the real world data (e.g., reliable test results). Toward better definition and providing the condition for using real world data, Eq. (17) is rewritten in Eq. (18) in terms of two positive control parameters $\left(a_{M}\right)$ and (b) (Ranjbaran et al., 2020b). Flexibility for translation and rotation of phenomenon functions in the $(1 \times 1)$ working space, which lets the experts to enforce their will, is provided by the form of phenomenon functions in Eq. (18) and selection of two control parameters from reliable real world data: 


$$
\left(S_{R} ; F_{R}\right)=\frac{\left(O^{b} ; a_{M} D^{b}\right)}{O^{b}+a_{M} D^{b}}
$$

To this end the system identification flag (state variable) and the mathematical basis for determination of the capacity is proposed, separately, in abstract form. Consequently the work is certain and universal, in a sense that it is independent of geometry, size, material property and etc. Therefore, it equally applies to all natural phenomena as change in systems. The system identification flag and the basic mathematical formulation are connected via the Persian Curve as follows.

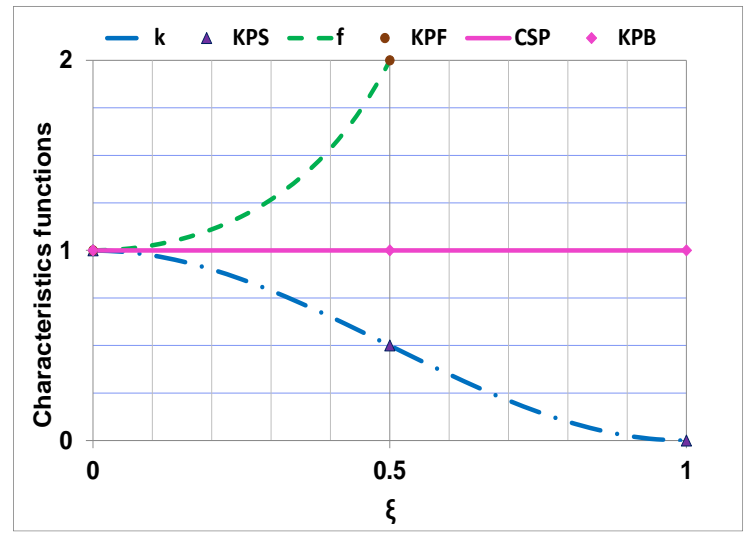

Fig. 3: Change of state philosophy basic equation

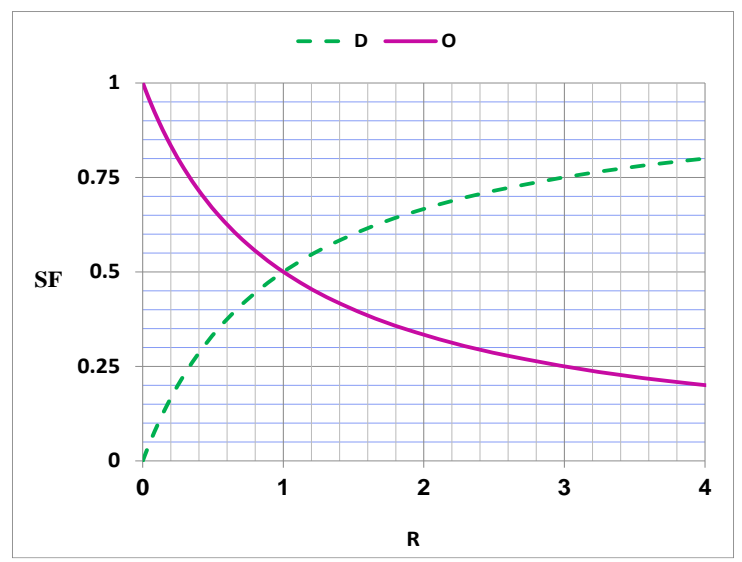

Fig. 4: State Functions versus the state ratio

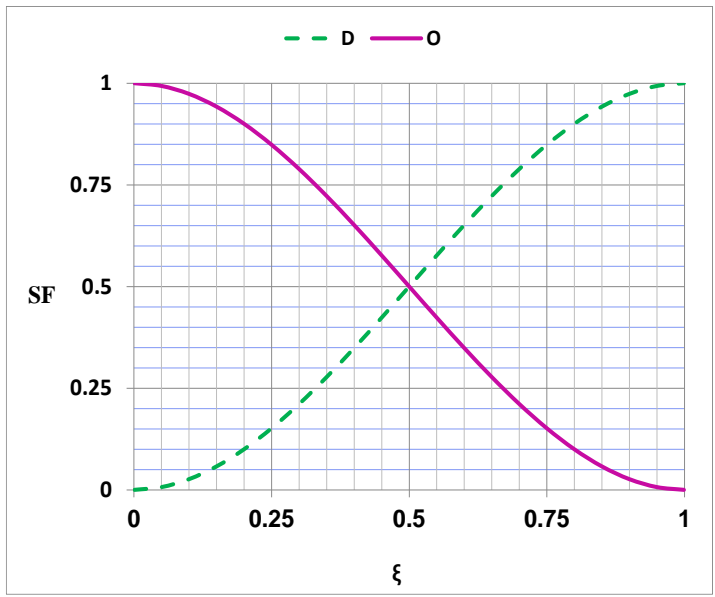

Fig. 5: State Functions versus the state variable 


\section{Persian Curve Birth}

The basic formulation for determination of capacity ratio is derived independent of real world data. In order to be able to apply the proposed formulation to natural phenomena (change in real systems), it must be connected to the system identification flag. This is done via construction of Persian Curve as follows. The phenomenon functions are mapped onto the real world data. The ratios at the beginning $\left(P_{O}\right)$ and at the termination $\left(P_{T}\right)$ of the phenomenon is selected from the reliable real world data, Fig. 6. For real natural phenomenon the $\left(S_{R}\right)$ is renamed as Shiraz curve $\left(P_{S}\right)$ and the $\left(F_{R}\right)$ is renamed as Fasa curve $\left(\mathrm{P}_{\mathrm{F}}\right)$ and collection of the two is called Persian Curve $\left(\mathrm{P}_{\mathrm{C}}\right)$ as defined in Eq. (19). Note that Eq. (19) for $\left(P_{O}=1\right.$ and $\left.P_{T}=0\right)$ and $\left(P_{O}=0\right.$ and $\left.P_{T}=1\right)$ converges to $\left(S_{R}\right)$ and $\left(F_{R}\right)$ in Eq. (18) respectively:

$$
P_{C}=\frac{P_{O} O^{b}+P_{T} a_{M} D^{b}}{O^{b}+a_{M} D^{b}}
$$

In comply with common practice in the literature, the derivative of phenomenon functions with respect to $(\xi)$ is called the Zahedan curve $\left(P_{Z}\right)$ as defined in Eq. (20), in which $\left(D^{(1)}\right)$ is derivative of $(D)$ with respect to $(\xi)$ :

$$
\begin{aligned}
& \left(F_{R} ; S_{R}\right)=\int_{(0 ; \xi)}^{(\xi ; 1)} P_{Z} d x \\
& P_{Z}=\frac{b a_{M} D^{b-1} O^{b-1} D^{(1)}}{\left(O^{b}+a_{M} D^{b}\right)^{2}}
\end{aligned}
$$

Note that, the $\left(P_{Z}\right)$ corresponds to the Probability Density Function (PDF) in the literature. To this end the formulation is complete. What remained is determination of the control parameters ( $a_{M}$ and $b$ ) for a real world data. This is done as follows. To prepare for simple and user friendly analysis, Eq. (19) is rewritten in Eq. (21), in which $\left(P_{C}\right)$ is coordinate of a point on real world data along the lifetime:

$$
a_{M}=\frac{a_{C}}{(D / O)^{b}}, a_{C}=\frac{P_{C}-P_{O}}{P_{T}-P_{C}}
$$

The aim is determination of ( $a_{M}$ and $b$ ) from nonlinear Eq. (21). Toward the aim, the coordinates of the middle point $(\mathrm{M})$ with $\left(D_{M} / O_{M}=1\right)$ and the next point $(\mathrm{N})$ (a point between $O, M$ ant $T$ ) are used in Eq. (21) to determine ( $a_{M}$ and $b$ ) from Eq. (22). Eq. (22) introduces the simplest method for analysis of nonlinear Eq. (21) and is the best tool for construction of a smooth curve to go through the key points $(O, N, M$ and $T)$ and with the extremum points at the ends, which is made with the expert will:

$$
\left(a_{N ; M} ; b\right)=\left(\frac{P_{N ; M}-P_{O}}{P_{T}-P_{N ; M}} ; \frac{\log \left(a_{N} / a_{M}\right)}{\log \left(D_{N} / O_{N}\right)}\right)
$$

The Key Points (KPS) are defined as the origin point $(\mathrm{O})$, the middle point $(\mathrm{M})$, the termination point $(\mathrm{T})$ and the next point $(\mathrm{N})$ (a point between the other three), in Eq. (23) and shown in Fig. 6 for both of increasing and decreasing data:

$$
\begin{array}{ccccc}
K P S & O & N & M & T \\
\xi & 0.0 & \xi_{N} & 0.5 & 1.0 \\
P & P_{O} & P_{N} & P_{M} & P_{T}
\end{array}
$$

\section{Unified Persian Curve}

Extensive review of literature (ANSI/AISC 360-16, 2016; AS 4100, 1998; Gardner, 2002; Ranawaka, 2006; Heva, 2009; Dolamune Kankanamge, 2010; Keerthan, 2010; Haidarali, 2011; Shahbazian, 2013; Cheng, 2015; Kucukler et al., 2015; Nguyen, 2017; Qiu, 2017; Szalai2017; Rasmussen, 2017; Ye, 2018; Imran et al., 2018), regarding real world data for failure of systems (Structures), led to the unified control parameters in Eq. (24) and the unified Persian curves in Eq. (25):

$$
\left(a_{M} ; b\right)=(2 ; 1)
$$

$\left(P_{S U} ; P_{F U} P_{Z U}\right)\left(\frac{1-0.8 D}{1+D} ; \frac{1.8 D}{1+D} ; \frac{2 D^{(1)}}{(1+D)^{2}}\right)$

In view of Eq. (25) the unified key points coordinates are defined in Eq. (26). The Persian Curve model is shown in Fig. 2, in a form to be comparable with the Monte Carlo model in Fig. 1:

$\begin{array}{ccccc}P C & O & N & M & T \\ \lambda & 0.000 & 0.750 & 1.500 & 3.000 \\ \xi & 0.000 & 0.250 & 0.500 & 1.000 \\ P_{F U} & 0.000 & 0.237 & 0.600 & 0.900 \\ P_{S U} & 1.000 & 0.763 & 0.400 & 0.100 \\ P_{Z U} & 0.000 & 1.686 & 1.364 & 0.000\end{array}$

\section{Comparison of the Persian Curve and the Monte Carlo Simulation}

Comparison of Eq. (6) and Eq. (19), that is the Monte Carlo curve (PMC) and the Persian Curve (PC), shown that both are system specific functions. Albeit the former is expensive and contains epistemic uncertainty, while the latter is cheap, simple and free of epistemic uncertainty. The idea behind the two are shown 
graphically in Fig. 1 and 2. respectively. More comparison of the results are included as follows.

The Persian curve is defined in terms of an abstract lifetime in a unit interval $(\xi \in[0,1])$. In real phenomena the lifetime is selected in a truncated interval $\left(\lambda \in\left[\lambda_{O}, \lambda_{T}\right]\right)$, where $\left(\lambda_{O}\right)$ is the origin lifetime and $\left(\lambda_{T}\right)$ is the termination lifetime. In order to use the Persian Curve for real world data, the $(\lambda)$ should be mapped onto $(\xi)$ as in Eq. (27):

$$
\begin{aligned}
& \lambda=(1-\xi) \lambda_{O}+\xi \lambda_{T} \\
& \xi=\left(\lambda-\lambda_{O}\right) /\left(\lambda_{T}-\lambda_{O}\right)
\end{aligned}
$$

In comply with the literature, the results of the Persian Curve is compared with that of the Monte Carlo Method in the following example.

Example 1: Iervolino et al. (2016; Baltzopoulos et al., 2017), developed a fragility curve for a six-story reinforced concrete moment resisting Frame $(\mathrm{BF})$, via using the SPO2FRAG software. Compare the (BF) with the Persian-Fasa-curve as the proposed fragility function. Note that the SPO2FRAG is developed based on the Monte Carlo Method.
Solution: Via hundreds of thousands of expensive nonlinear incremental dynamic analyses, the required data is prepared and the Fragility curve (BF) (a log-normal curve fitted on the data) is developed, which is scanned and shown in Fig. 7. The selected Key Points coordinates $(\mathrm{KPF})$ and the corresponding control parameters $\left(a_{M}\right)$ and (b) are expressed in Eq. (28) and Eq. (29):

$$
\begin{array}{ccccc}
K P F & O & N & M & T \\
\xi & 0.00 & 0.25 & 0.50 & 1.00 \\
P & 0.00 & 0.237 & 0.60 & 0.90 \\
& & & & \\
\lambda & \xi & a_{M} & b & \\
{[0,3]} & {[0,1]} & 2.00 & 1.00 &
\end{array}
$$

The unified Persian-Fasa-Curve (PFU), the unified Persian-Shiraz-curve (PSU) and the unified PersianZahedan-curve (PZU) from Eq. (25) are also shown in Fig. 7. Total agreement of the results in general and at the key points (KPF) in specific, is used as a flag for verification of the validity of the unified fragility curve. This close agreement is a powerful reason for recommending the (PC) as a replacement for the (MCS)

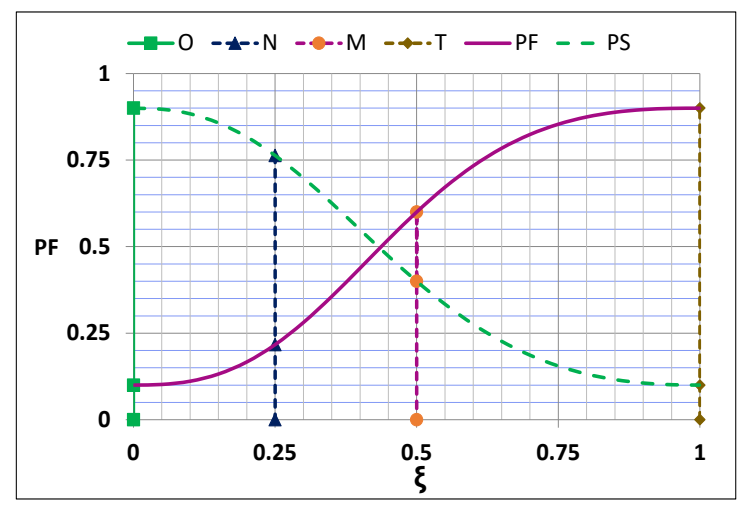

Fig. 6: Key points on persian-shiraz- and persian-fasa-curves

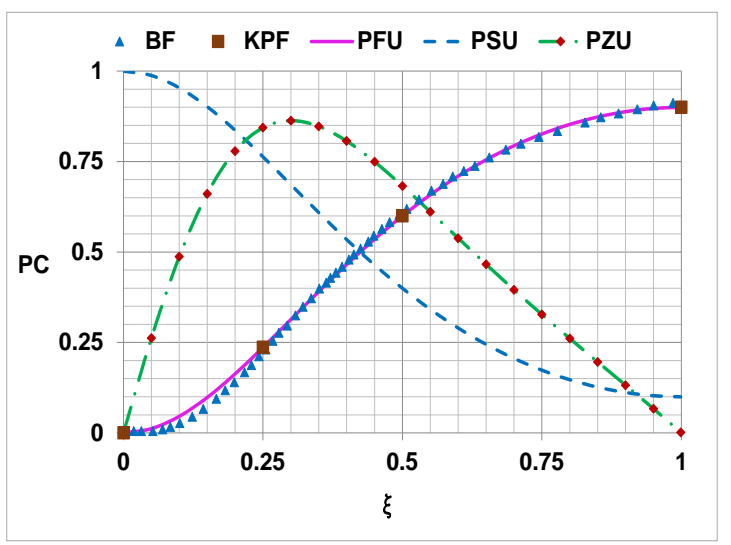

Fig. 7: Comparison of the (BF) and the persian curves 


\section{Conclusion}

The following conclusions are obtained from the present study:

- The Persian Curve is equal to the expected value of output in (MCS) with probability 1

- The Persian Curve is an excellent replacement for the Monte Carlo Simulation

- The Persian Curve is the best method for analysis of real world data

- The problems that were solved by the Monte Carlo Simulation is recommended to be resolved with the Persian Curve

- Replacement of the Monte Carlo Simulation by the Persian Curve is a revolution in human knowledge

\section{Acknowledgement}

Thanks to all members of our family, specially Zahra, who provided a calm state and condition, at the time of Corona at home, and encouraged writing of the paper. Note that the names of Persian curves were selected as the names of cities, Fasa, Shiraz and Zahedan in Iran, where the authors birthed, grown, educated and worked

\section{Author's Contributions}

Abdolrasoul Ranjbaran: Designed and contributed in writing the first draft and later corrections of the manuscript.

Mohammad Ranjbaran: Contributed in literature review, derivation of equations and engaged in the research team discussions.

Fatema Ranjbaran: Contributed in numerical analysis and engaged in the research team discussions.

\section{Ethics}

This article is original and contains unpublished material. The corresponding author confirms that all of the other authors have read and approved the manuscript and no ethical issues involved with declaration of no conflict of interest.

\section{References}

Alamri, A. S. N. A. (2020). Reliability analysis methods for power systems with substantial penetration of renewable generating resources (Doctoral dissertation, Georgia Institute of Technology). https://smartech.gatech.edu/handle/1853/62773

Albes, T. (2019). Kinetic Monte Carlo Simulations of Organic Solar Cells (Doctoral dissertation, Technische Universität München). https://mediatum.ub.tum.de/1444206
Anderson, T. L. (2017). Fracture mechanics: fundamentals and applications. CRC press. https://doi.org/10.1201/9781420058215

ANSI/AISC 360-16. (2016). Specification for structural steel buildings. ANSI/AISC 360-16. https://www.aisc.org/globalassets/aisc/publications/s tandards/a360-16-spec-and-commentary.pdf

Apostolopoulou, M. (2019). Fluid transport through porous media: A novel application of kinetic Monte Carlo simulations (Doctoral dissertation, UCL (University College London)). https://discovery.ucl.ac.uk/id/eprint/10088047/

AS 4100. (1998). Australian Standards. Steel structures. NSW, Australia: Standards Australia; 1998. https://www.saiglobal.com/pdftemp/previews/osh/as /as4000/4100/n4100.pdf

Badger, L. (1994). Lazzarini's lucky approximation of $\pi$. Mathematics Magazine, 67(2), 83-91. https://doi.org/10.1080/0025570X.1994.11996194

Baltzopoulos, G., Baraschino, R., Iervolino, I., \& Vamvatsikos, D. (2017). SPO2FRAG: software for seismic fragility assessment based on static pushover. Bulletin of Earthquake Engineering, 15(10), 43994425. https://doi.org/10.1007/s10518-017-0145-3

Berg, T. V. (2019). Combining analytical power system reliability assessment methods with Monte Carlo simulation (Master's thesis, NTNU). https://ntnuopen.ntnu.no/ntnuxmlui/bitstream/handle/11250/2625901/no.ntnu:insp era:40537039:35379393.pdf?sequence $=1$

Bhatia, B. S. (2020). Monte Carlo investigations of a high resolution Small Field-Of-View gamma camera (Doctoral dissertation, University of Leicester). https://leicester.figshare.com/articles/thesis/Monte_ Carlo_investigations_of_a_high_resolution_Small_ Field-Of-View_gamma_camera/12639341

Bolin, C. E. (2013). Iterative uncertainty reduction via Monte Carlo simulation: A streamlined life cycle assessment case study (Doctoral dissertation, Massachusetts Institute of Technology). https://dspace.mit.edu/handle/1721.1/82189

Bonate, P. L. (2001). A brief introduction to Monte Carlo simulation. Clinical pharmacokinetics, 40(1), 15-22. https://doi.org/10.2165/00003088-200140010-00002

Cheng, S. (2015). Fire performance of cold-formed steel sections. https://pearl.plymouth.ac.uk/handle/10026.1/3316

Cook, S. E., Mueller, E., \& Scheichl, R. (2013). Multi Level Monte Carlo Methods for Atmospheric Dispersion Modelling (Doctoral dissertation, University of Bath). https://researchportal.bath.ac.uk/files/187918997/SCoo kMPhilThesis_Final.pdf

Corbella, A. (2019). Statistical inference in stochastic/deterministic epidemic models to jointly estimate transmission and severity (Doctoral dissertation, University of Cambridge). https://www.repository.cam.ac.uk/handle/1810/294438 
Cosgrove, P. (2020). Numerical stability of Monte Carlo neutron transport and isotopic depletion for nuclear reactor analysis (Doctoral dissertation, University of Cambridge).

https://www.repository.cam.ac.uk/handle/1810/311429

Cumberworth, A. (2021). Monte Carlo simulation of DNA origami self-assembly (Doctoral dissertation, University of Cambridge). https://www.repository.cam.ac.uk/handle/1810/317057

Dash, M. (2020). Accurate excited states with quantum Monte Carlo: looking beyond conventions.

de Freitas, I. M. A. T. (2019). MOCAPIRA-Monte Carlo parallel implementation for reliability assessment. https://repositorioaberto.up.pt/bitstream/10216/121405/2/343986.pdf

Debrot, E. (2020). Applications of silicon dosimeters and Monte Carlo simulations for physical and biological dose quality assurance in particle therapy. https://ro.uow.edu.au/theses $1 / 795 /$

Diniz, G. S. (2020). Monte Carlo methods on low and high energy atmospheric phenomena. https://repositorio.unb.br/handle/10482/39672

Dolamune Kankanamge, N. (2010). Structural behaviour and design of cold-formed steel beams at elevated temperatures (Doctoral dissertation, Queensland University of Technology). https://eprints.qut.edu.au/33221/

Du, S. (2012). Monte Carlo Molecular Simulation with Isobaric-Isothermal and Gibbs-NPT Ensembles (Doctoral dissertation). https://repository.kaust.edu.sa/handle/10754/273072

Ead, R. (2020). Using Monte Carlo Simulation to Evaluate Performance of Forecasting Models in Project Control. https://era.library.ualberta.ca/items/ffcc7678-d6c6499f-9f97-94461a6184fb

Eagle, J. T. (2020). Monte Carlo characterisation of proton minibeams https://ir.canterbury.ac.nz/handle/10092/101690

Fadele, D. (2017). Reliability evaluation of a wind integrated power system with compressed air energy storage (Doctoral dissertation, University of Saskatchewan). https://harvest.usask.ca/handle/10388/8257

Feng, G. (2017). Efficient Reliability and Sensitivity Analysis of Complex Systems and Networks with Imprecise Probability (Doctoral dissertation, University of Liverpool). https://livrepository.liverpool.ac.uk/3009365/

Gardner, L. (2002). A new approach to structural stainless steel design. https://spiral.imperial.ac.uk/bitstream/10044/1/8862/ 1/L_Gardner-2002-PhD-Thesis.pdf
Goerdin, S. A. V. (2014). Application of Monte Carlo Simulation for risk-based decision making in MV distribution networks. https://repository.tudelft.nl/islandora/object/uuid:c09 bd3de-68a7-46ea-8a5b-b6b557429a93

Guijarro Gámez, G. (2020). Quantum Monte Carlo study of few-and many-body bose systems in one and two dimensions. https://upcommons.upc.edu/handle/2117/340966

Hahn, G. (2015). Statistical Methods for Monte-Carlo based Multiple Hypothesis Testing. https://core.ac.uk/download/pdf/77002206.pdf

Haidarali, M. R. (2011). Local and distortional buckling behaviour of cold-formed steel $\mathrm{Z}$ section beams. https://spiral.imperial.ac.uk/handle/10044/1/6992

Haqiqat, F., \& Müller, M. (2018, July). Analyzing the impact of knowledge and search in Monte Carlo Tree Search in Go. In Workshop on Computer Games (pp. 127-146). Springer, Cham. https://doi.org/10.1007/978-3-030-24337-1_7

Heva, Y. B. (2009). Behaviour and design of cold-formed steel compression members at elevated temperatures (Doctoral dissertation, Queensland University of Technology). https://eprints.qut.edu.au/29310/

Hochuli, R. (2016). Monte Carlo methods in quantitative photoacoustic tomography (Doctoral dissertation, UCL (University College London)). https://discovery.ucl.ac.uk/id/eprint/1507921/

Hou, W. (2018). Reliability assessment of HVdc systems using Monte Carlo simulation technique. https://mspace.lib.umanitoba.ca/handle/1993/33200

Huda, A. S. (2018). Multilevel Monte Carlo approach for estimating reliability of electric distribution systems (Doctoral dissertation). https://digital.library.adelaide.edu.au/dspace/hand le/2440/118116

Iervolino, I., Baltzopoulos, G., Vamvatsikos, D., \& Baraschino, R. (2016, June). SPO2FRAG v1. 0: software for PUSHOVER-BASED derivation of seismic fragility curves. In Proceedings of the VII European congress on computational methods in applied sciences and engineering, ECCOMAS, Crete Island, Greece (pp. 5-10). https://doi.org/10.7712/100016.2233.11553

Imran, M., Mahendran, M., \& Keerthan, P. (2018). Mechanical properties of cold-formed steel tubular sections at elevated temperatures. Journal of Constructional Steel Research, 143, 131-147. https://doi.org/10.1016/j.jcsr.2017.12.003

Keerthan, P. (2010). Shear behaviour and design of LiteSteel beams (Doctoral dissertation, Queensland University of Technology). https://doi.org/10.1201/b10571-129

Ketron, T. W. (2020). Monte Carlo Simulations on Resistive Switching Memristor Modeling (Doctoral dissertation, Rice University). https://scholarship.rice.edu/handle/1911/108343 
Kucukler, M., Gardner, L., \& Macorini, L. (2015). Flexural-torsional buckling assessment of steel beam-columns through a stiffness reduction method. Engineering Structures, 101, 662-676. https://doi.org/10.1016/j.engstruct.2015.07.041

Laengen, Ø. S. (2018). Application of Monte Carlo simulation to power system adequacy assessment (Master's thesis, NTNU). https://ntnuopen.ntnu.no/ntnuxmlui/handle/11250/2564804

Laosiritaworn, Y. (2002). Modelling the magnetic properties of thin metallic films using Monte-Carlo simulation (Doctoral dissertation, University of Warwick). https://ethos.bl.uk/OrderDetails.do?uin=uk.bl.ethos. 25 0093

Lefebvre, G. (2007). Practical Issues in Modern Monte Carlo Integration (Doctoral dissertation, McGill University Libraries). https://central.baclac.gc.ca/.item?id=TC-QMM-

103209\&op=pdf\&app=Library\&oclc_number $=8913$ 37063

Manohar, L. P. (2009). Reliability Assessment of a Power Grid with Customer Operated CHP Systems Using Monte Carlo Simulation. Masters Theses, 348. https://scholarworks.umass.edu/cgi/viewcontent.cgi? article $=1433 \&$ context $=$ theses

Mazhdrakov, M., Benov, D., \& Valkanov, N. (2018). The Monte Carlo method: engineering applications. ACMO Academic Press. ISBN-10: 6199068432

Mouawad, L. (2017). Monte Carlo simulations and a theoretical study of the damage induced by ionizing particles at the macroscopic scale as well as the molecular scale (Doctoral dissertation, Strasbourg). https://www.theses.fr/2017STRAE009

Murray, I. (2007). Advances in Markov chain Monte Carlo methods (Doctoral dissertation, University of London).

https://homepages.inf.ed.ac.uk/imurray2/pub/07thesi s/murray_thesis_2007.pdf

Nguyen, V. V. (2017). Direct Strength Method for the Design of Cold-Formed Steel Sections Under Localised Loading. https://ses.library.usyd.edu.au/handle/2123/17069

Nilakanta, H. (2020). Output Analysis of Monte Carlo Methods with Applications to Networks and Functional Approximation. https://conservancy.umn.edu/handle/11299/215058

Pakyuz-Charrier, E. J. (2018). Uncertainty is an Asset: Monte Carlo simulation for uncertainty estimation in implicit 3D geological modelling. https://doi.org/10.1071/ASEG2018abW10_2D

Parkinson, A. (2013). Modelling laser light propagation in thermoplastics using Monte Carlo simulations (Doctoral dissertation). https://qspace.library.queensu.ca/handle/1974/8327
Pollock, M. (2013). Some Monte Carlo methods for jump diffusions (Doctoral dissertation, University of Warwick). http://citeseerx.ist.psu.edu/viewdoc/download?doi=1 $0.1 .1 .449 .4972 \&$ rep $=$ rep $1 \&$ type $=$ pdf

Poole, T. (2014). Calculating derivatives within quantum Monte Carlo. https://core.ac.uk/download/pdf/76992603.pdf

Qiu, W. (2017). Beam-Column behaviour of concretefilled elliptical hollow sections. https://spiral.imperial.ac.uk/handle/10044/1/48035

Ranawaka, T. (2006). Distortional buckling behaviour of cold-formed steel compression members at elevated temperatures (Doctoral dissertation, Queensland University of Technology). https://eprints.qut.edu.au/16417/6/Thanuja_Ranawa ka_Thesis.pdf

Ranjbaran, A. (2010). Analysis of cracked members the governing equations and exact solutions. https://www.sid.ir/en/Journal/ViewPaper.aspx?ID=1 96258

Ranjbaran, A. (2012). Analysis of cracked members: free vibration, buckling, dynamic stability. Lap Lambert Academic Publishing Saarbrucken Germany. ISBN10: 978-3-659-20841-6

Ranjbaran, A. (2015). Buckling analysis of stiffened frames. NED University Journal of Research, 12(1), 29.

Ranjbaran, A., \& Ranjbaran, M. (2014). New finite-element formulation for buckling analysis of cracked structures. Journal of Engineering Mechanics, 140(5), 04014014. https://doi.org/10.1061/(ASCE)EM.19437889.0000734

Ranjbaran, A., \& Ranjbaran, M. (2016). State functions: the milestone of fracture. Archive of Applied Mechanics, 86(7), 1311-1324. https://doi.org/10.1007/s00419-015-1115-3

Ranjbaran, A., \& Ranjbaran, M. (2017a). State-based buckling analysis of beam-like structures. Archive of Applied Mechanics, 87(9), 1555-1565. https://doi.org/10.1007/s00419-017-1273-6

Ranjbaran, A., \& Ranjbaran, M. (2017b). State based damage mechanics. NED University Journal of Research, 14(1), 13-26.

Ranjbaran, A., \& Ranjbaran, M. (2017c). Innovative theory for the compliance computation in rotors. Scientia Iranica, 24(4), 1779-1788. https://doi.org/10.24200/sci.2017.4269

Ranjbaran, A., \& Rousta, A. (2009). A step toward happy ending to free vibration analysis of cracked members. NED University Journal of Research, 6(2), 113-123.

Ranjbaran, A., \& Rousta, H. (2013a). Interaction diagram for dynamic stability by Laplace transform. NED University Journal of Research, 10(1), 31-39.

Ranjbaran, A., \& Rousta, H. (2013b). Finite element analysis of cracked beams innovative weak form equations. NED University Journal of Research, $10(1), 39-47$. 
Ranjbaran, A., Hashemi, S., \& GHAFARIAN, A. (2008). A new approach for buckling and vibration analysis of cracked column. https://www.sid.ir/en/journal/ViewPaper.aspx?ID=1 14422

Ranjbaran, A., Ranjbaran, M., \& Baharvand, A. (2020a). A STEP TOWARD DEVELOPMENT OF GENERIC FRAGILITY CURVES. NED University Journal of Research, 17(3).

Ranjbaran, A., Ranjbaran, M., \& Ranjbaran, F. (2020b). Change of state philosophy \& Persian curves. LAP LAMBERT Academic Publishing, Germany, 2020, p. 225. ISBN-10: 978-620-2-68228-2.

Ranjbaran, A., Ranjbaran, M., \& Ranjbaran, F. (2020c). Building probability functions by Persian curves. International Journal of Structural Glass and Advanced Materials Research, 2020; 4(1); 225-232. https://doi.org/10.3844/sgamrsp.2020.225.232

Ranjbaran, A., Ranjbaran, M., \& Ranjbaran, F. (2020d). A reliable fracture mechanics. International Journal of Reliability, Risk and Safety: Theory and Application, 3(1), 1-15. https://doi.org/10.30699/IJRRS.3.1.1

Ranjbaran, A., Ranjbaran, M., \& Ranjbaran, F. (2020e). Building probability functions by Persian curve. International Journal of Structural Glass and Advanced Materials Research, 4(1); 225-232. https://doi.org/10.3844/sgamrsp.2020.225.232

Ranjbaran, A., Ranjbaran, M., \& Ranjbaran, F. (2020f). A reliable method of analysis for geotechnical data. International Journal of Structural Glass and Advanced Materials Research, 4(1); 276-293. https://doi.org/10.3844/sgamrsp.2020.276.293

Ranjbaran, A., Ranjbaran, M., \& Ranjbaran, F. (2021a). Building design rule for glass structures by Persian curve. International Journal of Structural Glass and Advanced Materials Research, 5(1); 1-13. https://doi.org/10.3844/sgamrsp.2021.1.13

Ranjbaran, A., Ranjbaran, M., \& Ranjbaran, F. (2021b). Development of a reliable failure assessment diagram by Persian curve. International Journal of Structural Glass and Advanced Materials Research, 5(1); 68-81. https://doi.org/10.3844/sgamrsp.2021.68.81

Ranjbaran, A., \& Ranjbaran, M. (2018). State based method for ultimate strength analysis of stiffened panels. Ned University Journal of Research, 15(1).

Ranjbaran, A., Rousta, H., \& Ranjbaran, M. (2013a). Dynamic stability of cracked columns; the stiffness reduction method. Scientia Iranica, 20(1), 57-64. https://doi.org/10.1016/j.scient.2012.11.005

Ranjbaran, A., Rousta, H., Ranjbaran, M. O., Ranjbaran, M. A., Hashemi, M., \& Moravej, M. T. (2013b). A necessary modification for the finite element analysis of cracked members detection, construction and justification. Archive of Applied Mechanics, 83(7), 1087-1096. https://doi.org/10.1007/s00419-0130736-7
Ranjbaran, A., Shokrzadeh, A. R., \& Khosravi, S. (2011). A new finite element analysis of free axial vibration of cracked bars. International Journal for Numerical Methods in Biomedical Engineering, 27(10), 1611-1621. https://doi.org/10.1002/cnm.1400

Rasmussen, K. (2017). Stability of thin-walled structural members and systems. https://ses.library.usyd.edu.au/handle/2123/18194

Rawlinson, D. (2015). Monte Carlo Simulations of an Anthropomorphic Phantom for Molecular Radiotherapy (MRT) with GATE (Doctoral dissertation, The University of Manchester (United Kingdom)). https://www.research.manchester.ac.uk/portal/files/545 68176/FULL_TEXT.PDF

Romano, P. K. (2013). Parallel algorithms for Monte Carlo particle transport simulation on exascale computing architectures (Doctoral dissertation, Massachusetts Institute of Technology). https://doi.org/10.1016/j.anucene.2012.06.040

Sánchez, L. G. G. (2016). Analysis of power distribution systems using a multicore environment (Doctoral dissertation, Universitat Politècnica de Catalunya). https://core.ac.uk/download/pdf/81577235.pdf

Schwarm, F. W. (2017a). Monte Carlo Simulation of Cyclotron Lines in Strong Magnetic Fields-Theory and Application. https://opus4.kobv.de/opus4fau/frontdoor/index/index/docId/8521

Schwarz, L. R. (2017b). Projector Quantum Monte Carlo Methods for Linear and Non-linear Wavefunction Ansatzes (Doctoral dissertation, University of Cambridge). https://doi.org/10.1103/PhysRevLett.118.176403

Shahbazian, A. (2013). Simplified thermal and structural analysis methods for cold-formed thin-walled steel studs in wall panels exposed to fire from one side. The University of Manchester (United Kingdom).

Sheridan-Methven, O. (2021). Nested multilevel Monte Carlo methods and a modified Euler-Maruyama scheme utilising approximate Gaussian random variables suitable for vectorised hardware and lowprecisions (Doctoral dissertation, University of Oxford). https://ora.ox.ac.uk/objects/uuid:c54891e5ad24-4520-9bfe-0eb1e6af7973

Sheridan-Methven, O. (2021). Nested multilevel Monte Carlo methods and a modified Euler-Maruyama scheme utilising approximate Gaussian random variables suitable for vectorised hardware and lowprecisions (Doctoral dissertation, University of Oxford). https://doi.org/10.3390/jmse9040380

Szalai, J. (2017). Complete generalization of the AyrtonPerry formula for beam-column buckling problems. Engineering Structures, 153, 205-223. https://doi.org/10.1016/j.engstruct.2017.10.031 
Unwin, H. J. T. (2018). Uncertainty quantification of engineering systems using the multilevel Monte Carlo method (Doctoral dissertation, University of Cambridge).

https://www.repository.cam.ac.uk/handle/1810/277877

Veach, E. (1998). Robust Monte Carlo methods for light transport simulation. Stanford University. https://search.proquest.com/openview/9235a026c14 $241 \mathrm{fecc} 3 \mathrm{~d} 849 \mathrm{e} 3 \mathrm{~d} 51568 \mathrm{e} / 1$ ?pqorigsite $=$ gscholar $\& \mathrm{cbl}=18750 \&$ diss $=\mathrm{y}$

Wang, C. (2018). Applications of Monte Carlo methods in studying polymer dynamics (Doctoral dissertation, University of Reading). http://centaur.reading.ac.uk/80310/

Wang, C. (2021). Estimation of time-dependent reliability of aging structures under correlated load and autocorrelation in resistance deterioration. Applied Mathematical Modelling, 94, 272-284. ISBN-10: 978-3-030-62504-7.

Wang, L. (2015). Methods in Monte Carlo computation, astrophysical data analysis and hypothesis testing with multiply-imputed data (Doctoral dissertation). https://dash.harvard.edu/handle/1/17463134

Webster, S. (2019). Improved Monte Carlo Simulations of Massive Quarks (Doctoral dissertation, Durham University). http://etheses.dur.ac.uk/12954/

Welding, J. (2020). Sequential Monte Carlo methods for epidemic data (Doctoral dissertation, Lancaster University). https://eprints.lancs.ac.uk/id/eprint/141011/

Wijesinghe, P. (2011). Biased Monte Carlo methods for efficient simulation of communication systems. https://researchdirect.westernsydney.edu.au/islandor a/object/uws:11188/

Ye, J., Mojtabaei, S. M., Hajirasouliha, I., Shepherd, P., \& Pilakoutas, K. (2018). Strength and deflection behaviour of cold-formed steel back-to-back channels. Engineering Structures, 177, 641-654. https://doi.org/10.1016/j.engstruct.2018.09.064

Zhang, L. (2019). A Monte Carlo Model of Pitting Corrosion of Underground Power Transmission Cable (Doctoral dissertation, University of Leicester).

https://leicester.figshare.com/articles/thesis/A_Mont e_Carlo_Model_of_Pitting_Corrosion_of_Undergro und_Power_Transmission_Cable/10235165

Zhao, S. (2016). Advanced Monte Carlo simulation and machine learning for frequency domain optical coherence tomography (Doctoral dissertation, California Institute of Technology). https://thesis.library.caltech.edu/9597/

Zio, E. (2013). Monte carlo simulation: The method. In The Monte Carlo simulation method for system reliability and risk analysis (pp. 19-58). Springer, London. https://doi.org/10.1007/978-1-4471-4588-2.

\section{List of Symbols}

A:

$a_{M}:$

$a_{N}:$

$b$ :

CSP:

$D$ :

$d:$

$E$ :

$f(x, \theta)$ :

$F$ :

$F_{y}$ :

$f_{S}$ :

$f_{C}$ :

$F_{S}$ :

$f_{S S}$ :

$F_{R}$ :

$\left(F_{R}\right.$ and $\left.S_{R}\right)$ :

$f_{O}(O)$ :

$h(\xi, \theta)$ :

$I_{i}$ :

$k_{S}:$

$k_{C}$ :

$k_{S S}$ :

$K_{P F}$ :

$K_{P S}$ :

$K_{P B}$ :

$L$ :

$L$ :

$\lambda:$

$\lambda=\frac{L}{r} \sqrt{\frac{F_{y}}{\pi^{2} E}}:$

$\lambda_{O}:$

$\lambda_{T}$ :

$\mu$ :

$m$ :

$M:$

$M_{C S}$ :

$n$ :

$n$ :

$N$ :

$O$ :

$O$ :

$O_{i}$ :

$O_{D}$ :

$P(A)$ :

$P_{C}$ :

$P_{O}$ :

$P_{N}$ :

$P_{M C}$ :

$P_{M}$ :

$P_{T}$ :

$P_{F U}$ :
Event of needle crossing line

Control parameter at $M$

Control parameter at $N$

Control parameter (power)

Change of State Philosophy

Destination function

Distance between parallel lines

Initial modulus

Joint probability density function

Force

Yield limit

System flexibility

Change flexibility

Dimensioned flexibility

Survived flexibility

Failure function

Phenomenon functions

Probability density function

System characteristics function

Random input variable

System stiffness

Change stiffness

Survived stiffness

Key points on Failure curve

Key points on Survive curve

Key points on CSP curve

Effective length

Needle length

Lifetime parameter

Relative slenderness ratio

Lifetime origin

Lifetime termination (end)

Average of random output

Number of crossing event

Middle point

Mont Carlo Simulation

Number of random variables

Number of needle throw

Next point

Origin (start) point.

Origin function

Random output variable

Ordered designation

Probability of A

Persian curve (s)

Origin point ordinate

Next point ordinate

Mont Carlo Simulation function.

Middle point ordinate

End point ordinate

Unified Persian-Failure function 
Abdolrasoul Ranjbaran et al. / International Journal of Structural Glass and Advanced Materials Research 2021, Volume 5: 234.246 DOI: $10.3844 /$ sgamrsp.2021.234.246

$\begin{array}{llll}P_{S U}: & \text { Unified Persian-Survive function } & S_{R}: & \text { Survive function } \\ P_{Z U}: & \text { Unified Persian-distribution function } & S F=(\mathrm{D} \text { and } \mathrm{O}): & \text { State functions } \\ P_{Z}: & \text { Persian-Distribution function } & T: & \text { Termination (end) point } \\ P_{F}: & \text { Persian-Failure function } & \theta: & \text { Angle between line and needle } \\ P_{S}: & \text { Persian-Survive function } & \xi: & \text { State variable } \\ P C=(P F \text { and } P S): & \text { Persian curves } & \psi: & \text { Displacement } \\ r: & \text { Effective radius of gyration } & x: & \text { Working parameter } \\ R: & \text { State ratio } & & \end{array}$

